\title{
Keinicer konkret
}

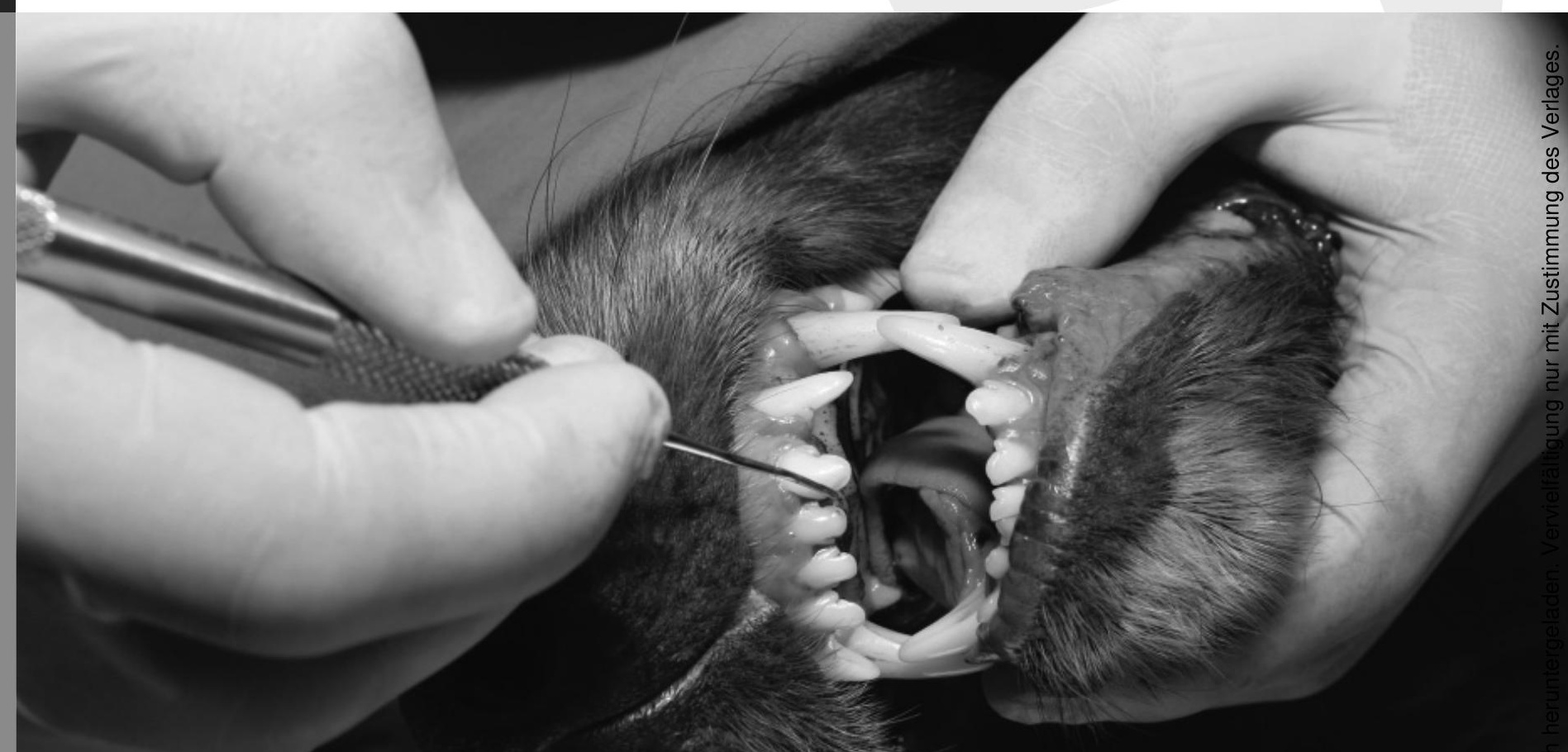

Jahresverzeichnis 2010 
Herausgeber:

Dr. med. vet. Ulrike Arnold, Stuttgart

(Schriftleitung)

Prof. Dr. med. vet. Michaele Alef, Leipzig

TA Maurice Bob, Berlin

Verlag:

Enke Verlag in MVS Medizinverlage

Stuttgart GmbH \& Co. KG

Oswald-Hesse-Str. 50, 70469 Stuttgart,

Fax: 0711/8931-706

Chefredaktion (v.i.S.d.P.):

Dr. med. vet. Ulrike Arnold

Tel.: 07 11/89 31-743,

Fax: 07 11/89 31-706

ulrike.arnold@enke.de

Anzeigenleitung:

MVS Mediaservice, Ilona Reiser,

Tel.: $0711 / 8931-713$,

Fax: 07 11/89 31-706

ilona.reiser@medizinverlage.de
Marketing:

Katrin Proß, Tel.: 07 11/8931-726,

Fax: 07 11/8931-748

katrin.pross@medizinverlage.de

Abonnementservice

und Probeabonnement:

KundenServiceCenter Zeitschriften, Tel.: 07 11/89 31-321, Fax: -422,

aboservice@thieme.de

\section{Produktion:}

Christel Idalinya, Tel.: 07 11/89 31-716, Fax: 07 11/89 31-748

christel.idalinya@medizinverlage.de

Satz:

Sommer Buch- und Offsetdruckerei, Feuchtwangen

Druck und Weiterverarbeitung:

Kliemo AG, Eupen (Belgien)

Erscheinungsweise: 6-mal im Jahr, davon 4-mal mit Beilage team.konkret - der Zeitschrift für Tiermedizinische Fachangestellte zzgl. einem kleintier.konkret Sonderheft zu den Baden-Badener Fortbildungstagen kleintier.konkret.

Bezugspreise 2010: Jahresabo inkl. kleintier.konkret Sonderheft und 4-mal jährlich Beilage team.konkret: 119,00€, Studierende: $49,90 €$ (bei Vorlage der Immatrikulationsbescheinigung), jeweils zzgl. Versandkosten: Inland 12,50€, Europa 20,90€, Übersee 31,90€. Preis für ein Einzelheft 25,40€.

Alle Preise inkl. MwSt. Der Abonnementpreis wird im Voraus in Rechnung gestellt. Die Bezugsdauer verlängert sich jeweils um ein Jahr, wenn keine Abbestellung zum 30. September erfolgt

Manuskripte: Der Verlag haftet nicht für Manuskripte, die unverlangt eingereich werden. Grundsätzlich werden nur solche Manuskripte angenommen, die vorher weder im Inland noch im Ausland veröffentlicht worden sind. Die Manuskripte dürfen auch nicht gleichzeitig anderen Blättern zum Abdruck angeboten werden. Mit der Annahme des Manuskriptes zur Veröffentlichung überträgt der Verfasser für die Dauer der gesetzlichen Schutzfrist (§ 64 UrhG) dem Verlag die ausschließlichen Verwertungsrechte im Sinne der $\S \S 15$ ff des UrhG für alle Auflagen/Updates, insbesondere das Recht zur Vervielfältigung in gedruckter Form, in elektronischen Medienformen (Datenbanken, Online-Netzsysteme, Internet, CD-ROM, DVD etc.) sowie zur Übersetzung und Weiterlizenzierung.

Copyright: Die Zeitschrift und alle in ihr enthaltenen einzelnen Beiträge und Abbildungen sind für die Dauer des Urheberrechts geschützt. Jede Verwertung außerhalb der engen Grenzen des Urheberrechtsgesetzes ist ohne Zustimmun des Verlages unzulässig und strafbar. Das gilt insbesondere für Vervielfältigungen, Übersetzungen, Mikroverfilmungen und die Einspeicherung und Verarbeitung in elektronischen Systemen.

Wichtiger Hinweis: Für Angaben über Dosierungsanweisungen und Applikationsformen kann vom Verlag keine Gewähr übernommen werden. Jeder Benutzer ist angehalten, durch sorgfältige Prüfung der Beipackzettel der verwendeten Präparate und gegebenenfalls nach Konsultation eines Spezialisten festzustellen, ob die dort gegebene Empfehlung für Dosierungen oder die Beachtung von Kontraindikationen gegenüber der Angabe in dieser Zeitschrift abweicht. Eine solche Prüfung ist besonders wichtig bei selten verwendeten Präparaten oder solchen, die neu aufden Markt gebracht worden sind. Jede Dosierung oder Applikation erfolgt auf eigene Gefahr des Benutzers. Autoren und Verlag appellieren an jeden Benutzer, ihm etwa auffallende Ungenauigkeiten dem Verlag mitzuteilen.

Hinweis für unsere Leser: Der Verlag ist um eine zuverlässige Zustellung der abonnierten Zeitschrift äußerst bemüht. Gelegentlich versäumen Abonnenten nach einem Umzug, ihre neue Anschrift zu nennen. In diesen Fällen hilft die Deutsche Post AG, die neue Anschrift dem Verlag mitzuteilen. Abonnenten, die mit diesem Vorgehen nicht einverstanden sind, werden gebeten, dies dem Verlag zu schreiben.

Thieme-connect: Ausgewählte Inhalte der kleintier.konkret (ab dem Jahrgang 2007), der team.konkret (neu ab Ausgabe $1 / 2010$ ), sowie den vormals erschienenen Zeitschriften Tierarzthelferin.konkret (Ausgabe 1/2008 - 4/2009) und team spiegel $(1 / 2003-4 / 2009)$ stehen online in Thieme-connect zur Verfügung. Internetseite: www.thieme-connect.de/ejournals.

Der Zugang ist für persönliche Abonnenten im Preis enthalten. Bei Fragen wenden Sie sich bitte per E-mail an das Thieme-connect Helpdesk: helpdesk@thieme-connect.de, Tel.: 0711/8931-420. Über kostenpflichtige Zugangsmöglichkeiten und Lizenzen für Institutionen (Bibliotheken, Kliniken, Firmen, etc.) informiert Sie gerne das Sales-Team, E-mail: sales@thieme-connect.de, Tel.: 0711/8931-407. 


\begin{tabular}{|c|c|c|c|c|c|}
\hline Heft 1 & (Februar & 2010) = Seite & 3 & & 3 \\
\hline Heft 2 & (April & 2010) = Seite & 3 & - & \\
\hline Heft 3 & (Juni & 2010) = Seite & 3 & - & \\
\hline Heft 4 & (August & 2010) = Seite & 3 & - & \\
\hline Heft 5 & (Oktober & 2010) = Seite & 3 & - & \\
\hline Heft 6 & (Dezember & 2010) = Seite & 3 & - & 3 \\
\hline Heft S 1 & (März & 2010) = Seite & 3 & - & \\
\hline
\end{tabular}

CVE

19 Fuchs, Simone, Anne Schmidt, Gerhard Oechtering, Michaele Alef, Ingmar Kiefer Ultraschalldiagnostik der Nebennieren bei Hund, Katze und Frettchen -Möglichkeiten und Grenzen

16 Peters, Stefanie Arzneimittelreaktionen an der Haut - Klinik, Diagnose und Therapie

\section{Forum}

26 Posch, Birte

Die Vermessung des Kaninchens

\section{Heimtiere}

13 Baran, Kerstin

Kastration beim männlichen Degu - Narkoseregime und Operationstechnik

20 Bochmann, Monika

Präovulatorische Legenot bei Echsen - Diagnostik und Therapie

18 Kölle, Petra

Schildkröten - Der Panzer als Spiegel der Gesundheit

18 Müller, Kerstin

Schmerztherapie bei Heimtieren - Erfahrungen zum Einsatz von Opioidanalgetika

13 Pees, Kathrin

Überwinterung von Fischen - Häufige Probleme vor und nach der Winterruhe

12 Pees, Michael Herpesviren bei Landschildkröten - Symptomatik, Diagnose und Therapie

16 Pees, Michael Schimmelpilzmykose bei Vögeln - Therapie-Update

29 Schöne, Richard, Volker Schmidt, Margit Sachse, Ronald Schmäschke

Laufsittiche - Befiederungsstörung durch bisher unbekannte Milbe

\section{Hund}

3 Kessler, Martin

c-KIT \& Co - Häufig gestellte Fragen zum Mastzelltumor des Hundes

3 Koch, Daniel

Magendrehung beim Hund - Ein Update

3 Schroll, Sabine

Im-Kreis-Drehen, Schwanz einfangen und Co - Obsessiv-kompulsive Störungen beim Hund

8 Straubinger, Reinhard K., Nikola Pantchev

Die Lyme-Borreliose-Impfung beim Hund - kontrovers diskutiert
Hund und Katze

11 Alef, Michaele, Susanne Pfeifer Inhalationsnarkose mit Isofluran oder Sevofluran - Wie lässt sich das Risiko von Nebenwirkungen reduzieren?

14 Alef, Michaele, Ferdinand von Praun Präoperatives Laborscreening - Sinn oder Unsinn?

3 Dillitzer, Natalie, Sylviavon Rosenberg Fütterungstipps zum BARFen - So vermeiden Sie Fehlernährungen

8 Eickhoff, Markus

Parodontitis - Parodontale Bakteriämie verursacht Organerkrankungen

10 Lutz, Hans, Regina Hofmann Impfung individuell

20 Mrozek, Margund Vektorübertragene Krankheiten, Parasitosen und Zoonosen - aktuell und praxisnah

3 Nolte, Ingo, Patrick Wefstaedt

Antibiotikabehandlung bei Durchfallerkrankungen von Hund und Katze - Sinn oder Unsinn?

15 Schnieder, Thomas

Wurmbefall - Sachgerechte Beratung Schritt für Schritt 8 Schnyder, Manuela

Lungenwürmer - Relevanz und Maßnahmen in der Kleintierpraxis

8 Schroll, Sabine Phyto-Psychopharmaka für den Einsatz bei Hund und Katze - Indikationen und Dosierung

12 Truyen, Uwe Impfung individuell

9 Winkels, Philipp, Jan Wieser Intestinale Invagination - Ursache, Therapie, Prophylaxe

\section{Katze}

3 Bösing, Britta

Alfaxalon-Injektionsanästhesie bei der Hauskatze - Klinische Anwendbarkeit und Praktikabilität

24 Rade, Claudia, Ralf Tobias

Diätetik bei herzkranken Katzen - Gibt es einen „katzenspezifischen" Ansatz?

3 Robertson, Sheilah A.

Akute Schmerzen bei Katzen erkennen - Ein Leitfaden für die Praxis

\section{Laborgeräte}

24 Niesterock, Christian, Jens-Christian Rudnick, Claudia Haarstrick, Michaele Alef, Ingmar Kiefer Sonografische Lymphknotendiagnostik - Welche Möglichkeiten bieten moderne Ultraschallgeräte?

22 Zohmann, Andreas, Reinhold Scharwey, Ellen Preutenborbeck

Die Interferenzstrom-Regulationstherapie - Elektromedizin der (erstaunlich) anderen Art

\section{Newsletter, Forschung und Industrie}

28 Mougeot, Isabelle

Diätetisches Stressmanagement - Die anxiolytische Wirkung von Alpha-Casozepin und Co. 
Praxismanagement

32 Bienefeld, Birte

Kombinierte Präventionsleistungen in der Tierarztpraxis - So erweitern Sie Ihr Leistungsangebot

26 Blättner, Antje Customer Relationship Management - Kundenpflege in der Tierarztpraxis

32 Blättner, Antje Wie sprechen Sie mit Ihren Kunden? Rezeption - die Schlüsselstelle jeder Praxis

31 Dornbusch, Hans-Ludwig

Aufwendungen des Tierarztes für einen Pkw - Besteuerung im Jahre 2010

30 Dornbusch, Hans-Ludwig

Personalkosten und Steuern in der Tierarztpraxis 2010

- Änderungen durch das Wachstumsbeschleunigungsgesetz

32 Dornbusch, Hans-Ludwig

Steuerliche Berücksichtigung von Vorsorgeaufwendungen - Neuregelung 2010

32 Menges, Christine

Der letzte Hund - Hundehaltung für alte Menschen

Rundumgeschaut

26 Deininger, Elke

Animal Hoarding - Was ist das? 


\section{A}

Alef, Michaele 11, 14, 19, 24

\section{B}

Baran, Kerstin 13

Bienefeld, Birte 32

Blättner, Antje 26, 32

Bochmann, Monika 20

Bösing, Britta 3

\section{D}

Deininger, Elke 26

Dillitzer, Natalie 3

Dornbusch, Hans-Ludwig 30, 31, 32

$$
\text { E }
$$

Eickhoff, Markus 8

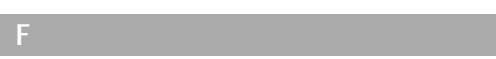

Fuchs, Simone 19

$$
\mathrm{H}
$$

Haarstrick, Claudia 24

Hofmann, Regina 10

$$
\text { K }
$$

Kessler, Martin 3

Kiefer, Ingmar 19, 24

Koch, Daniel 3

Kölle, Petra 18

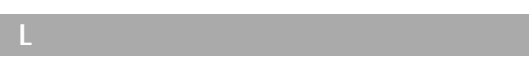

Lutz, Hans 10

\section{M}

Menges, Christine 32

Mougeot, Isabelle 28

Mrozek, Margund 20

Müller, Kerstin 18

\section{N}

Niesterock, Christian 24

Nolte, Ingo 3

$$
0
$$

Oechtering, Gerhard 19

\section{P}

Pantchev, Nikola 8

Pees, Kathrin 13

Pees, Michael 12, 16

Peters, Stefanie 16

Pfeifer, Susanne 11

Posch, Birte 26

Preutenborbeck, Ellen 22

\section{R}

Rade, Claudia 24

Robertson, Sheilah A. 3

Rosenberg, Sylviavon 3

Rudnick, Jens-Christian 24

$$
\text { S }
$$

Sachse, Margit 29

Scharwey, Reinhold 22

Schmäschke, Ronald 29

Schmidt, Anne 19

Schmidt, Volker 29

Schnieder, Thomas 15

Schnyder, Manuela 8

Schöne, Richard 29

Schroll, Sabine 3, 8

Straubinger, Reinhard K. 8

\section{$\mathrm{T}$}

Tobias, Ralf 24

Truyen, Uwe 12

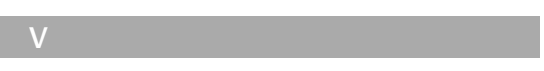

von Praun, Ferdinand 14

\section{W}

Wefstaedt, Patrick 3

Wieser, Jan 9

Winkels, Philipp 9

\section{Z}

Zohmann, Andreas 22 